patches. ${ }^{4}$ In suspected patients, a thorough history of allergic reactions to balloons, gloves, barium enema, and other latex devices should be taken. The gold standard in the diagnosis is skin-prick testing in patients with localized symptoms and latex-specific IgE antibody assessment in cases of systemic symptoms. ${ }^{5}$ However, the wear and/or use test and the patch test are the alternative diagnostic tests. ${ }^{4}$ The most effective approach for the management of latex allergy is personal and environmental avoidance by considering hypoallergic gloves. ${ }^{4}$

Recommendations for the prevention of allergic reactions to gloves are summarized in Fig. 1. Urticaria can be treated with antihistamines and the elimination of the antigen. $\mathrm{H}_{1,2}$ Blockers can be used before coming into contact with latex devices; however, latex avoidance is superior to this protocol. ${ }^{4}$

Plastic gloves, considered as hypoallergenic polyvinylchloride (PVC) gloves, are also used among HCWs. Contact allergic reaction to PVC has also been reported as a result of allergy to many additives used in these gloves, including carba mix, mercaptobenzothiazole (MBT), thiuram mix, mixed dialkyl thioureas, and black rubber mix. ${ }^{5}$ Allergic contact dermatitis has been reported in numerous case reports. Lesions may also become generalized in some patients. A patch test can be used to confirm the diagnosis. Topical corticosteroids are considered as the best choice of treatment; however, patients should be advised to use other types of gloves, ${ }^{5}$ although allergic contact dermatitis may coexist with immediate hypersensitivity to Latex. ${ }^{5}$

Nitrile, neoprene, and polyurethane are also used in plastic gloves. Hand dermatitis has been reported with these types of materials. The patch test is recommended in suspicious cases. Application of topical and oral steroids can mitigate the symptoms but the benefits should be weighed against the risks of side effects. $^{6}$

Glove-related hand urticaria should also be considered as a differential diagnosis; it is caused by dermographism upon the application of the glove. Pain, burning, and pruritus in the affected area, and systemic symptoms such as fever are not present in glove-related hand urticaria. Further, nitrile gloves are more likely to cause this phenomenon because they are rigid and less flexible.
Some types of powder used in gloves have been associated with an increased risk of skin roughness due to altering glove $\mathrm{pH} .{ }^{8}$ Glove powder has been reported to cause allergic reactions, and hand eczema has been reported to decrease significantly after using powder-free gloves. ${ }^{9}$ Thus, the use of powder-free gloves is recommended in the current pandemic situation. HCWs are also encouraged to wear double gloves when handling COVID-19 patients' airways, blood, urine, and other body fluids. The outer glove should be the first equipment to be removed. ${ }^{2}$

\section{Acknowledgments.}

Financial support. No financial support was provided relevant to this article.

Conflicts of interest. All authors report no conflicts of interest relevant to this article.

\section{References}

1. Kwon KT, Ko J-H, Shin H, Sung M, Kim JY. Drive-through screening center for COVID-19: a safe and efficient screening system against massive community outbreak. J Korean Med Sci 2020;35.

2. Chen X, Shang Y, Yao S, Liu R, Liu H. Perioperative care provider's considerations in managing patients with the COVID-19 infections. Transl Perioper Pain Med 2020;7:216-223.

3. Alenius H, Turjanmaa K, Palosuo T. Natural rubber latex allergy. Occupat Environ Med 2002;59:419-424.

4. Taylor JS, Erkek E. Latex allergy: diagnosis and management. Dermatol Ther 2004;17:289-301.

5. Bolognia JL, Schaffer JV, Cerroni L. Dermatology, 4th ed. London: Elsevier Health Sciences; 2018.

6. Dooms-Goossens A, Bruze M, Buysse L, Fregert S, Gruvberger B, Stals H. Contact allergy to allergy to allyl glycidyl ether present as an impurity in 3-glycidyl-oxypropyl-trimethoxysilane, a fixing additive in silicone and polyurethane resins. Contact Dermatitis 1995;33:17-19.

7. Hawkey S, Abdul Ghaffar S. Glove-related hand urticaria: an increasing occupational problem among healthcare workers. Br J Dermatol 2016;174:11371140 .

8. Brehler R, Voss W, Muller S. Glove powder affects skin roughness, one parameter of skin irritation. Contact Dermatitis 1998;39:227-230.

9. Edelstam G, Arvanius L, Karlsson G. Glove powder in the hospital environment-consequences for healthcare workers. Int Arch Occupat Environ Health 2002;75:267-271.

\title{
Pharynx gargle samples are suitable for SARS-CoV-2 diagnostic use and save personal protective equipment and swabs
}

\author{
Monika Malecki $\mathrm{PhD}^{1}$, Jessica Lüsebrink $\mathrm{PhD}^{2}$, Stefanie Teves $\mathrm{BSc}^{1}$ and Andreas F. Wendel $\mathrm{MD}^{1}$ (1) \\ ${ }^{1}$ Institute of Hygiene, Cologne Merheim Medical Centre, University Hospital of Witten/Herdecke, Cologne, Germany and ${ }^{2}$ Institute of Pathology, Cologne Merheim \\ Medical Centre, University Hospital of Witten/Herdecke, Cologne, Germany
}

To the Editor-First described in China in December 2019, novel coronavirus (SARS-CoV-2), which causes coronavirus disease 2019 (COVID-19), has spread globally. Europe is now an

\footnotetext{
Author for correspondence: Andreas F. Wendel, E-mail: wendela@kliniken-koeln.de Cite this article: Malecki M, et al. (2021). Pharynx gargle samples are suitable for SARS-CoV-2 diagnostic use and save personal protective equipment and swabs. Infection Control \& Hospital Epidemiology, 42: 248-249, https://doi.org/10.1017/ ice. 2020.229
}

epicenter of the COVID-19 pandemic. To bring the epidemic under control, laboratory testing for SARS-CoV-2 is essential to diagnose and isolate infected people and subsequently trace their contacts. Public health agencies recommend rapid initial testing by polymerase chain reaction (PCR) from upper respiratory (nasopharyngeal or oropharyngeal) specimens in ambulatory patients. ${ }^{1,2}$ SARS-CoV-2 replicates in the throat and the lung, and throat samples have a sufficient sensitivity in the first episode 
of the disease. ${ }^{3}$ However, healthcare workers (HCWs) obtaining the swab must wear personal protective equipment (PPE set: respirator, eye protection, gloves and gowns) because coughing might be induced by triggering the gag reflex of the person to be sampled.

In this pandemic, a foreseeable shortage of PPE and an acute lack of flocked swabs occurred in our hospital. Hence, we decided to collect pharynx gargle samples as an upper respiratory tract specimen (also known as oral rinse or throat wash in the literature). Pharynx gargle specimens can be obtained without close contact between the patient and the healthcare worker. Furthermore, pharynx gargle samples are easy to collect and sample the same anatomic region as throat swabs. Pharynx gargle samples are also an established method for the molecular detection of common respiratory infections, as well as in children. ${ }^{4,5}$ However, to our knowledge, only a few studies have assessed this type of specimen for the diagnosis of viral respiratory diseases. Bennet et $\mathrm{al}^{5,6} \mathrm{dem}$ onstrated that gargle samples were more sensitive in the detection of viral respiratory pathogens, and some evidence shows that gargle samples are suitable for SARS-CoV-2 diagnosis. ${ }^{6,7}$ Saliva collected by gargling has already been investigated for determining the viral load of SARS-CoV-2. ${ }^{8}$ In addition, gargle samples were successfully used in the first SARS epidemic for RNA detection and antigen testing. ${ }^{9,10}$

In March 2020, during the preparations for the first wave of infections, we installed examination units for HCWs in all 3 hospitals of our institution. HCWs were asked to come to the desk if they showed respiratory symptoms or if they had unprotected contact to a COVID-19 patient or SARS-CoV-2-positive HCW. We established the following workflow: The HCW approaches the desk, where a Plexiglas pane has been installed, and keeps a distance of at least $1.5 \mathrm{~m}$. When symptomatic, the HCW wears a face mask. If a test is deemed necessary, he or she is instructed to provide a pharynx gargle sample in an empty room nearby equipped with a test kit (specimen container, $10 \mathrm{~mL}$ normal saline). After sampling (gargling time, 10-30 s), the closed container is left in the room. The throat wash is quickly transferred to a biosafety 2 laboratory and is subjected to a reverse transcription PCR for SARS-CoV-2 detection (RealStar SARS-CoV-2 RT-PCR Kit, Altona Diagnostics, Germany). On a regular basis, windows are opened in the sampling room, and contact sites are disinfected after each visit. During the whole procedure, no additional special PPE or swabs are needed.

From mid-March until April 20, $924 \mathrm{HCW}$ sere tested at least once, and 26 samples were positive $(2.8 \%)$. Due to the limited number of PCR reagents and swabs, we examined only a very limited number $(\mathrm{n}=5)$ of paired specimens (throat swab and gargle sample taken within 24 hours) in our hospital. We have observed 1 discrepant result (ie, throat swab negative and gargle sample positive) so far. At the same time, we saved at least 225 PPE sets (conservative calculation of 3 sets per day and per hospital over a period of 25 work days) and 1,000 swabs.

Of course, this approach can only be used if the person being tested is able to gargle. Patients from whom a gargle sample cannot be obtained (eg, dysphagia, dementia or infants) should be swabbed. Gargle samples might only be manageable for laboratories if there are low numbers of specimens. The gargle sample container is bigger than a swab; thus, it might cause problems with packaging or take too much space in a safety cabinet. Finally, in some countries national guidelines do not allow gargle sampling.

In conclusion, self-collected gargle samples are easy to take, noninvasive, material saving, and safe for healthcare workers. Nevertheless, more preanalytic data and comparative studies are needed at different stages of COVID-19.

Acknowledgments. We thank the laboratory team of the Institute of Pathology and the Institute of Hygiene for routine diagnostics. We also thank the team that installed the examination unit, especially Ina Dombrowski.

Financial support. No financial support was provided relevant to this article.

Conflicts of interest. All authors report no conflicts of interest relevant to this article.

\section{References}

1. Interim guidelines for collecting, handling, and testing clinical specimens from persons for coronavirus disease 2019 (COVID-19). Centers for Diesase Control and Prevention. https://www.cdc.gov/coronavirus/2019$\mathrm{nCoV} / \mathrm{lab} /$ guidelines-clinical-specimens.html, Published 2020. Accessed April 22, 2020.

2. Laboratory testing for coronavirus disease (COVID-19) in suspected human cases. World Health Organization website. https://www.who.int/ publications-detail/laboratory-testing-for-2019-novel-coronavirus-insuspected-human-cases-20200117. Published 2020. Accessed April 22, 2020.

3. Wolfel R, Corman VM, Guggemos W, et al. Virological assessment of hospitalized patients with COVID-2019. Nature 2020. doi: 10.1038/ s41586-020-2196-x.

4. Morikawa S, Hiroi S, Kase T. Detection of respiratory viruses in gargle specimens of healthy children. J Clin Virol 2015;64:59-63.

5. Bennett S, MacLean A, Gunson R. Verification of Cepheid Xpert Xpress $\mathrm{Flu} / \mathrm{RSV}$ assay for use with gargle samples, sputa and endotracheal secretions. J Hosp Infect 2019;101:114-115.

6. Bennett S, Davidson RS, Gunson RN. Comparison of gargle samples and throat swab samples for the detection of respiratory pathogens. J Virol Methods 2017;248:83-86.

7. Saito M, Adachi E, Yamayoshi S, et al. Gargle lavage as a safe and sensitive alternative to swab samples to diagnose COVID-19: a case report in Japan. Clin Infect Dis 2020. Apr 2 [Epub ahead of print]. doi: 10.1093/ $\mathrm{cid} /$ ciaa377.

8. To KK, Tsang OT, Leung WS, et al. Temporal profiles of viral load in posterior oropharyngeal saliva samples and serum antibody responses during infection by SARS-CoV-2: an observational cohort study. Lancet Infect Dis 2020;20:565-574.

9. Liu IJ, Chen PJ, Yeh SH, et al. Immunofluorescence assay for detection of the nucleocapsid antigen of the severe acute respiratory syndrome (SARS)associated coronavirus in cells derived from throat wash samples of patients with SARS. J Clin Microbiol 2005;43:2444-2448.

10. Wang WK, Chen SY, Liu IJ, et al. Detection of SARS-associated coronavirus in throat wash and saliva in early diagnosis. Emerg Infect Dis 2004;10: 1213-1219. 